\title{
Magnetic breakdown in a normal-metal-superconductor proximity sandwich
}

\author{
Alban L. Fauchère and Gianni Blatter \\ Theoretische Physik, Eidgenössische Technische Hochschule, CH-8093 Zürich, Switzerland
}

(April 2, 2017)

\begin{abstract}
We study the magnetic response of a clean normal-metal slab of finite thickness in proximity with a bulk superconductor. We determine its free energy and identify two (meta-)stable states, a diamagnetic one where the applied field is effectively screened, and a second state, where the field penetrates the normal-metal layer. We present a complete characterization of the first order transition between the two states which occurs at the breakdown field $H_{b}(T)$, including its spinodals, the jump in the magnetization, and the latent heat. The bistable regime terminates at a critical temperature $T_{\text {crit }}$ above which the sharp transition is replaced by a continuous cross-over. We compare the theory with experiments on normal-superconducting cylinders.
\end{abstract}

PACS $74.50+\mathrm{r}, 75.30 . \mathrm{Kz}$

The superconducting proximity effect in a normal metal adjacent to a superconductor has received a revived interest in the past decade [1]. Among the fundamental equilibrium problems, the magnetic response of normalmetal-superconductor (NS) structures deserves particular interest. Experiments have demonstrated the nontrivial screening properties of these hybrid structures, exhibiting a hysteretic magnetic breakdown at finite fields [2.4] as well as a presently unexplained re-entrance in the magnetic susceptibility at low temperatures [5]. The investigated samples have typical dimensions comparable to the coherence length $\xi_{N}$ of the normal metal, attributing a key role to the quantum coherence of the electrons coupled to the macroscopic phase of the superconductor.

The self-consistent study of the screening currents in a NS sandwich within the framework of the GinsburgLandau (GL) equation was carried out a long time ago by the Orsay group [6]. Their work has provided the first understanding of the non-linear field phenomena such as the magnetic breakdown. However, in the proximity effect, the GL equations are at their limit of validity and their use is restricted to the dirty limit. The quasi-classical Green's function technique [7] allows to describe the clean limit using the Eilenberger equations $[8]$ and to generalize the dirty limit results using the Usadel equations [9]. Zaikin was the first to derive the magnetic response of a normal-metal slab of finite thickness connected to a bulk superconductor along these lines [10]. Most notably, he found a non-local screening behavior in the clean limit linear response which has an appealing similarity to the one found in superconductors of the Pippard type. The applied (static) magnetic field was found to be over-screened, the magnetic induction changing sign inside the normal layer. Using numerical methods, Belzig et al. 11] have investigated the non-linear field regime of these equations and found two (meta-)stable mean-field solutions in both the clean and the dirty limit. In this work, we determine the $H-T$ 'phase' diagram shown in
Fig. 11 of the normal metal layer in the clean limit, where the bistable regime is particularly extended. In thermodynamic equilibrium, we find a magnetic breakdown at $H_{b}(T)$, which is a first order transition separating the phase of diamagnetic screening from the phase of magnetic field penetration.

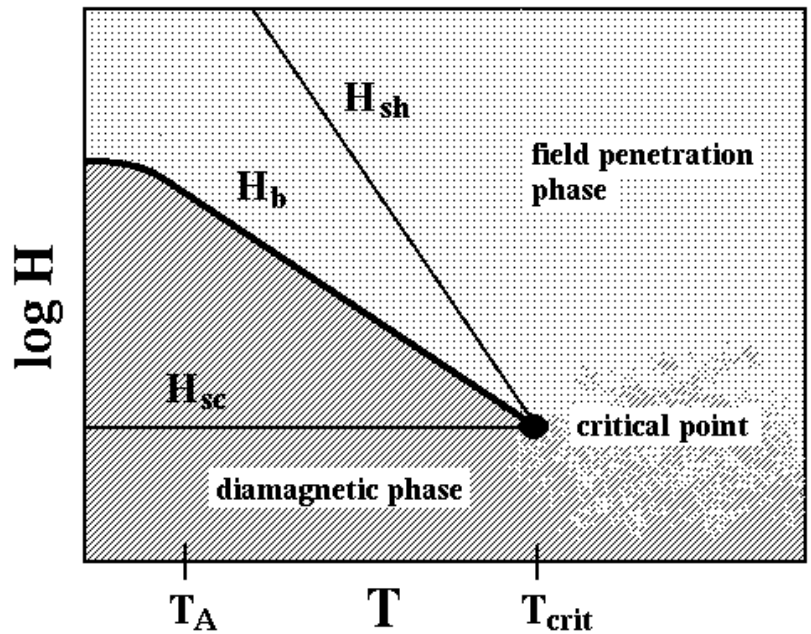

FIG. 1. $H-T$ phase diagram of the normal metal slab of thickness $d$. The breakdown field $H_{b}(T) \sim \Phi_{\circ} / \lambda_{N}(T) d$ marks the first order transition between the diamagnetic and the field penetration phase $\left(\lambda_{N}(T)\right.$ denotes the penetration depth, $\Phi_{\circ}$ the superconducting flux unit). The critical point at the intersection of the spinodals $H_{s c}(T) \sim \Phi_{\circ} / d^{2}$ and $H_{s h}(T) \sim \Phi_{\circ} / \lambda_{N}^{2}(T)$ separates the first order transition for $\lambda_{N}(T) \ll d$ from the continuous cross-over at large temperature $\left(\lambda_{N}(T) \gg d\right)$.

Mota et al. [12.13] have recently investigated the magnetic response of metallic cylinders with a superconducting core. Their data, which is not described by the results of the GL equations, was claimed to be characteristic for the ballistic limit [13]. This has motivated us to derive the analytic dependence of the clean limit expression for 
the breakdown field $H_{b}$ on temperature $T$ and thickness $d$ of the normal layer and to compare it to the experiment. From the free energy of the normal layer, which allows us to identify the two (meta-)stable states, we determine the spinodals, the thermodynamic breakdown field $H_{b}(T)$ and find the jumps in magnetization and entropy at the transition. Furthermore, we obtain a critical temperature which marks the upper limit of the bistable regime (see Fig. 1). Finally, we work out the signatures of the non-locality in the ballistic regime as they show up in the magnetic susceptibility $\chi$ and compare them with the experimental data. The following discussion is divided into four sections, the analysis of the constitutive relations (Sec. I), the solution of the magnetostatic problem (Sec. II), the determination of the breakdown field from the free energy (Sec. III), and finally the comparison with experiment (Sec. IV).

\section{CONSTITUTIVE RELATIONS}

The quasi-classical Green's function technique provides an appropriate description of a metal with nearly spherical Fermi surface. In a finite magnetic field, the vector potential $\mathbf{A}(\mathbf{x})$ can be included as a phase factor along unperturbed trajectories, provided the dimensions of the normal metal are smaller than the Larmor radius $\left(r_{L}=p_{F} c / e H\right.$, cyclotron radius of an electron traveling at Fermi velocity). In the ballistic limit, the quasiclassical $2 \times 2$ matrix Green's function $\hat{g}_{\omega_{n}}\left(\mathbf{x}, \mathbf{v}_{F}\right)$ satisfies the Eilenberger equation [8] $\left(e=|e|, \hbar=k_{B}=c=1\right)$,

$$
\begin{aligned}
& -\left(\mathbf{v}_{F} \cdot \nabla\right) \hat{g}_{\omega_{n}}\left(\mathbf{x}, \mathbf{v}_{F}\right)= \\
& {\left[\left(\omega_{n}+i e \mathbf{v}_{F} \cdot \mathbf{A}(\mathbf{x})\right) \hat{\tau}_{3}+\Delta(\mathbf{x}) \hat{\tau}_{1}, \hat{g}_{\omega_{n}}\left(\mathbf{x}, \mathbf{v}_{F}\right)\right],}
\end{aligned}
$$

where the mean-field order parameter $\Delta$ provides an offdiagonal potential $\left(\hat{\tau}_{i}\right.$ denote the Pauli matrices, $\omega_{n}=$ $(2 n+1) \pi T$ are the Matsubara frequencies, $[\cdot, \cdot]$ is the commutator). We have excluded elastic scattering processes by assuming $T \gg 1 / \tau_{e l}$.

We consider a normal metal slab of thickness $d$ on top of a bulk superconductor as shown in the inset of Fig. 1 . The vector potential $\mathbf{A}=(0, A(x), 0)$ describes a magnetic field $\mathbf{B}=(0,0, B(x))$ applied parallel to the surface, which induces screening currents $\mathbf{j}=(0, j(x), 0)$. We make the following idealizations in the description of the NS sandwich: The superconducting order parameter follows a step function $\Delta(x)=\Delta \theta(-x)(\Delta$ real $)$, no attractive interactions being present in the normal layer. We assume a perfect NS interface as well as specular reflection at the normal-vacuum boundary.

In the subsequent analysis we restrict our attention to the magnetic response of the normal layer. In the proximity effect, the macroscopic coherence of the superconducting condensate induces correlated electron-hole pairs in the normal layer through the process of Andreev reflection. The basic process consists of an electron traveling forward and a hole traveling backward along a quasi-classical trajectory as shown in Fig. 2 (at discrete energies, bound Andreev states are found along these trajectories). In the presence of a magnetic field, the area enclosed by the trajectory (see Fig. 2) is threaded by the flux

$$
\Phi(a, \vartheta, \varphi)=\oint \mathbf{A}(\mathbf{x}) \cdot d \mathbf{x}=2 \tan \vartheta \cos \varphi \int_{0}^{d} A(x) d x,
$$

which can be expressed through the integral $a=$ $\int_{0}^{d} A(x) d x$ times a geometric factor due to the inclination of the trajectory (the spherical angles $\vartheta$ and $\varphi$ parameterize the direction of the trajectory). The current carried along a trajectory depends on the phase factor $2 \Phi(a, \vartheta, \varphi) / \Phi_{\circ}$ acquired by the propagation of both the electron and the hole along the Andreev loop, and we arrive at an intrinsic non-local current-field dependence $j(a)$. The total current is determined by the sum over the currents along the quasi-classical trajectories,

$$
j_{y}(x)=\frac{i e m p_{F}}{\pi} T \sum_{\omega_{n}>0}\left\langle v_{y} \operatorname{Tr}\left[\hat{\tau}_{3} \hat{g}_{\omega_{n}}\left(x, \mathbf{v}_{F}\right)\right]\right\rangle
$$

(the brackets $\langle\ldots\rangle$ denote the average over the angles $\vartheta$, $\varphi$ ), from which we reproduce the expression first derived by Zaikin [10],

$$
j(a)=\int_{0}^{\pi / 2} d \vartheta \int_{0}^{\pi / 2} d \varphi j(\vartheta, \varphi, \Phi(a, \vartheta, \varphi)),
$$

where $\left(\alpha_{n}=2 \omega_{n} d / v_{F} \cos \vartheta\right)$

$$
\begin{aligned}
& j(\vartheta, \varphi, \Phi(a, \vartheta, \varphi))=-\frac{2 e p_{F}}{\pi^{2}} T \sum_{\omega_{n}>0} \sin ^{2} \vartheta \cos \varphi \\
& \times \frac{\Delta^{2} \sin 2 \pi \Phi / \Phi_{\circ}}{\left(\omega_{n} \cosh \alpha_{n}+\sqrt{\omega_{n}^{2}+\Delta^{2}} \sinh \alpha_{n}\right)^{2}+\Delta^{2} \cos ^{2} \pi \Phi / \Phi_{\circ}} .
\end{aligned}
$$

Note that $j$ is independent of $x$. The induced currents for each trajectory depend only on the flux $\Phi$ modulo the superconducting flux quantum $\Phi_{\circ}=\pi \hbar c / e$, reflecting gauge invariance. At small fields $\left(a / \Phi_{\circ} \ll 1\right)$, the current response is diamagnetic for all trajectories and the proximity effect produces screening currents in the normal metal. As the field increases to $a / \Phi_{\circ} \sim 1$, some of the more extended trajectories produce paramagnetic currents, since the reduced flux $\Phi \in\left[-\Phi_{\circ} / 2, \Phi_{\circ} / 2\right]$ they enclose becomes negative, and the net diamagnetic current response is reduced. As we reach large fields $\left(a / \Phi_{\circ} \gg 1\right)$, the Andreev levels become mutually dephased due to a uniform distribution of the reduced flux. The associated currents are randomly dia- or paramagnetic and the net 
current vanishes. Note that the proximity effect, i.e., the existence of the Andreev levels is not destroyed in this limit, leading to a finite kinetic energy of the currents induced by the magnetic field.

\section{MAGNETOSTATICS}

Owing to the independence of $j$ on $x$, the Maxwell equation $-\partial_{x}^{2} A(x)=4 \pi j$ and the constitutive equation (任) combined with the boundary conditions [14] $A(x=0)=0$ and $\partial_{x} A(x=d)=H$ can be given a formal solution. We arrive at a parabolic dependence for $A(x)=H x+4 \pi j(a) x\left(d-\frac{x}{2}\right)$ parameterized by $a=\int_{0}^{d} A(x) d x$, which in turn is determined through the self-consistency condition

$$
a=\frac{H d^{2}}{2}+\frac{4 \pi}{3} j(a) d^{3} .
$$

The total magnetization $\mathcal{M}$ (per unit surface) is defined by

$$
4 \pi \mathcal{M}=\int_{0}^{d} d x\left(\partial_{x} A(x)-H\right)=2 \pi j(a) d^{2} .
$$

Eq. (6) contains the essential physics of the problem: For small fields $(a \rightarrow 0)$, the current $j \approx-3 H / 8 \pi d$ linearly suppresses the magnetic induction on the geometric length scale $d$ to the value $B(0) \rightarrow-H / 2$ at the NS boundary. The magnetic induction is thus over-screened and assumes an opposite sign at the NS interface. A closer look shows that the current is given by the linear response expression

$$
j\left(a / \Phi_{\circ} \ll 1\right) \approx-\frac{1}{4 \pi \lambda_{N}^{2}(T) d} a,
$$

which depends on penetration depth $\lambda_{N}(T) \ll d$ to be derived below (Eq. (16p). When inserted back into (6), the vector potential is found to be strongly suppressed to $a \approx 3 H \lambda_{N}^{2}(T) / 2$, and we obtain a consistent diamagnetic solution (i.e., $a / \Phi_{\circ} \ll 1$ ) for fields up to $H<\Phi_{\circ} / \lambda_{N}^{2}$. At large fields, the current vanishes $(j \rightarrow 0)$ and the magnetic field penetrates the normal layer. From Eq. (6) we find $a \approx H d^{2} / 2$, consequently this metallic behavior is expected down to magnetic fields $H>\Phi_{\circ} / d^{2}$, as follows from the condition $a / \Phi_{\circ} \gg 1$ for the Andreev levels to be dephased. With $\Phi_{\circ} / d^{2} \ll \Phi_{\circ} / \lambda_{N}^{2}$ the diamagnetic and field penetration solution coexist in the regime $\Phi_{\circ} / d^{2}<H<\Phi_{\circ} / \lambda_{N}^{2}$. These simple estimates for the limits of the bistable regime elucidate the numerical data of Belzig et al. 11].

\section{THERMODYNAMICS}

In the phase diagram of Fig. 1 the upper and lower bounds of the bistable regime found from the above mean-field analysis are identified with the spinodals of the transition, the super-cooled field $H_{s c} \sim \Phi_{o} / d^{2}$ and the super-heated field $H_{s h} \sim \Phi_{o} / \lambda_{N}^{2}(T)$. In the thermodynamic equilibrium, a magnetic breakdown occurs at an intermediate field, connecting the diamagnetic regime to the field penetration regime by a first order transition. In the following, we determine this breakdown field and the associated entropy and magnetization jump from the free energy.

The energy (per unit surface) of the currents $j(x)=$ $-\delta F / \delta A(x)$ is obtained via an integration over the nonlinear current expression,

$$
\begin{aligned}
F(a) & =-\int_{0}^{a} j\left(a^{\prime}\right) d a^{\prime} \\
& =\frac{p_{F}^{2}}{\pi^{2}} T \sum_{\omega_{n}>0} \int_{0}^{\pi / 2} d \vartheta \int_{0}^{\pi / 2} d \varphi \sin \vartheta \cos \vartheta \\
\log & \frac{\left(\omega_{n} \cosh \alpha_{n}+\sqrt{\omega_{n}^{2}+\Delta^{2}} \sinh \alpha_{n}\right)^{2}+\Delta^{2}}{\left(\omega_{n} \cosh \alpha_{n}+\sqrt{\omega_{n}^{2}+\Delta^{2}} \sinh \alpha_{n}\right)^{2}+\Delta^{2} \cos ^{2} \pi \Phi / \Phi_{\circ}}
\end{aligned}
$$

$F(a)$ describes the difference in free energy between the metal layer under proximity and in the normal state. $F(a)$ is a monotonous and strictly positive function, reflecting the absence of condensation energy in the normal layer, and expresses the cost of the induced proximity effect lying in the kinetic energy of the currents induced by the vector potential. The free energy $\mathcal{F}(T, \mathcal{M})$ is constructed by adding the electro-magnetic field energy and subtracting the vacuum field contribution,

$$
\mathcal{F}(T, \mathcal{M})=F(a)+\int_{0}^{d} d x\left(\frac{\left(\partial_{x} A(x)\right)^{2}}{8 \pi}-\frac{H^{2}}{8 \pi}\right) .
$$

We do not include the condensation energy and the kinetic energy of the screening currents in the superconductor. The field dependent term of the condensation energy might in fact be of the order of the free energy in the normal layer and would be expected to produce numerical corrections in the results, which are not accounted for by our idealized choice of the order parameter $\Delta(x)=\Delta \theta(-x)$. The kinetic energy of the screening currents $\sim H^{2} \lambda$ may be neglected.

After a Legendre transformation, we obtain the Gibb's free energy

$$
\begin{aligned}
\mathcal{G}(T, H) & =F(T, \mathcal{M})-\mathcal{M} H \\
& =F(a)+\int_{0}^{d} d x \frac{\left(\partial_{x} A(x)-H\right)^{2}}{8 \pi} .
\end{aligned}
$$

The field term in Eq. (11) describes the work necessary to expel the magnetic field. The extrema of the free energy $\mathcal{G}$ with respect to $a$ reproduce the equation of state 
(6). Fig. 2 shows the free energy $\mathcal{G}(H)$ as obtained from the parameterization of $\mathcal{G}$ and $H$ through $a$ [15]. The breakdown field $H_{b}(T)$ is determined by the intersection of the free energies $\mathcal{G}$ of the two (meta-)stable solutions. We note that this procedure is equivalent to the Maxwell construction in the magnetization curve $\mathcal{M}=-\partial \mathcal{G} / \partial H$ of Fig. 2 .

In the following, we consider the free energy (9) in the two temperature limits $T=0$ and $T_{A} \ll T \leq \Delta$ and obtain $\left(T_{A}=v_{F} / 2 \pi d\right)$,

$$
\begin{aligned}
F_{T=0}(a) \approx & \frac{p_{F}^{3}}{4 \pi^{3} d m} \int_{0}^{\pi / 2} d \vartheta \int_{0}^{\pi / 2} d \varphi \sin \vartheta \cos ^{2} \vartheta \\
& \left\{\arctan \left[\tan \pi \Phi / \Phi_{\circ}\right]\right\}^{2} \\
F_{T \gg T_{A}}(a) \approx & \frac{4 p_{F}^{2} T}{\pi^{2}} \gamma^{2}(T, \Delta) \int_{0}^{\pi / 2} d \vartheta \int_{0}^{\pi / 2} d \varphi \sin \vartheta \cos \vartheta \\
& \exp \left(-\frac{2 T}{T_{A} \cos \vartheta}\right) \sin ^{2} \pi \Phi / \Phi_{\circ} .
\end{aligned}
$$

The finite value of the superconducting gap $\Delta$ is accounted for by the dimensionless parameter

$$
\gamma(T, \Delta)=\Delta /\left(\sqrt{\Delta^{2}+(\pi T)^{2}}+\pi T\right)<1
$$

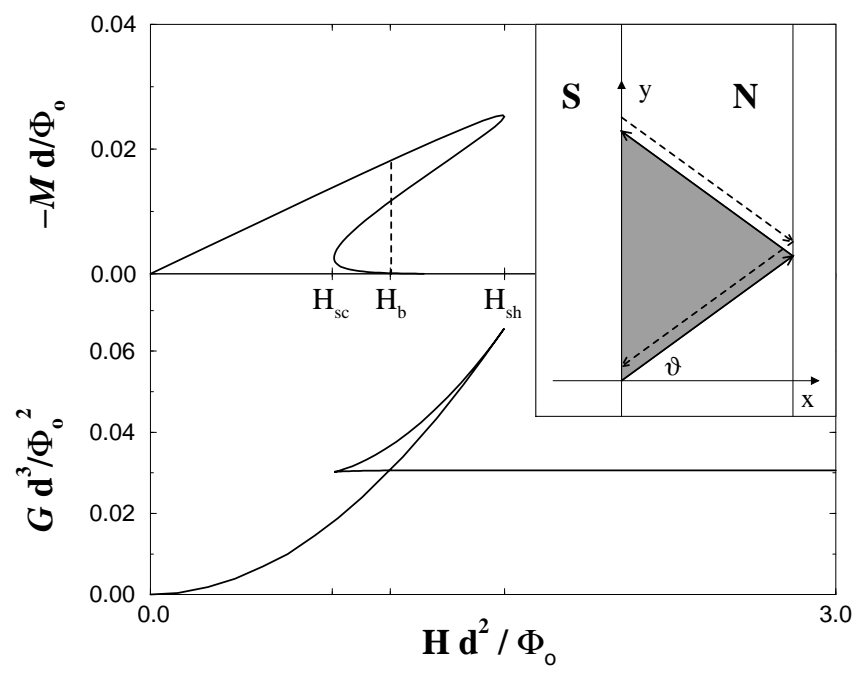

FIG. 2. Magnetization $\mathcal{M}(H)$ and free energy $\mathcal{G}(T, H)$ at a temperature $T=1.5 T_{A}\left(T_{A}=v_{F} / 2 \pi d\right)$. The representation is universal in the thickness $d$. The small and large field branches represent (meta-)stable solutions describing the diamagnetic and field penetration phases, which overlap in the field interval $H_{s c}<H<H_{s h}$. The Maxwell construction determines the first order transition between the phases at the breakdown field $H_{b}$ (dashed line). Inset: Cross-section of the normal-metal slab in contact with the bulk superconductor. The quasi-classical electron-hole trajectory at angles $\vartheta, \varphi=0$ encloses a flux that enters as a phase factor in the wavefunction.

The free energies of the two (meta-)stable stable states can be approximated by their asymptotic forms in the limits $a \rightarrow 0$ and $a \rightarrow \infty$. In the diamagnetic regime, the expansion in $a / \Phi_{\circ}$ up to quadratic order of (12) and (13) provide the result

$$
F(a) \approx \frac{a^{2}}{8 \pi \lambda_{N}^{2}(T) d}
$$

Eq. (15) is valid both in the low and high temperature limits using the penetration depth $1 / \lambda_{N}^{2}(0) \equiv 1 / \lambda_{N}^{2}=$ $\left(4 \pi n e^{2} / m\right)$ at $T=0$ and

$$
\frac{1}{\lambda_{N}^{2}(T)} \approx \frac{1}{\lambda_{N}^{2}} \gamma^{2}(T, \Delta) \frac{6 T_{A}}{T} e^{-2 T / T_{A}}
$$

for $T \gg T_{A}$. Note that the derivative $j=-\partial F / \partial a$ applied to Eq. (15) produces the linear response constitutive relation of Eq. (8). The Gibb's free energy follows from Eqs. (10) and (15), using the solution of the Maxwell equations,

$$
\mathcal{G}\left(a \ll \Phi_{\circ}\right) \approx \frac{3}{32 \pi} H^{2} d .
$$

Eq. (17) is dominated by the magnetization work necessary to expel the field, which is parametrically larger (by $\left.\left(d / \lambda_{N}(T)\right)^{2}\right)$ than the kinetic energy of the currents.

In the field penetration regime we approximate the free energy by its asymptotic value at $a \rightarrow \infty$. In this limit we replace the strongly oscillating functions of $\Phi$ in (12) and (13) by their average value $\left\langle(\arctan \tan \Phi)^{2}\right\rangle=\pi^{2} / 12$ and $\left\langle\sin ^{2} \Phi\right\rangle=1 / 2$ and obtain

$$
\begin{aligned}
\mathcal{G}_{T=0}\left(a \gg \Phi_{\circ}\right) & \approx \frac{1}{384 \pi} \frac{\Phi_{o}^{2}}{\lambda_{N}^{2} d}, \\
\mathcal{G}_{T \gg T_{A}}\left(a \gg \Phi_{\circ}\right) & \approx \frac{3}{16 \pi^{3}} \gamma^{2}(T, \Delta) \frac{\Phi_{o}^{2}}{\lambda_{N}^{2} d} e^{-2 T / T_{A}} .
\end{aligned}
$$

The magnetization energy vanishes in this limit. The corrections to the free energy (18) are of relative order $\left(\Phi_{\circ} / a\right)^{2}$.

The magnetic breakdown field $H_{b}(T)$ is determined by the intersection of the two asymptotics of the free energy $\mathcal{G}$ given by Eqs. (17) and (18),

$$
\begin{aligned}
H_{b}(T=0) & \approx \frac{1}{6} \frac{\Phi_{o}}{\lambda_{N} d}, \\
H_{b}\left(T \gg T_{A}\right) & \approx \frac{\sqrt{2}}{\pi} \gamma(T, \Delta) \frac{\Phi_{o}}{\lambda_{N} d} e^{-d / \xi_{N}(T)} .
\end{aligned}
$$

We note three important features of this result: The temperature dependence is a simple exponential with the exponent $d / \xi(T)=T / T_{A}$, where $\xi_{N}(T)=v_{F} / 2 \pi T$ denotes the normal metal coherence length. The amplitude of the breakdown field scales inversely proportional to the thickness of the normal layer, $H_{b} \sim 1 / d$. In the limit $T \rightarrow 0$ the magnetic breakdown field saturates to a value 
which is suppressed by the universal factor $\pi / 6 \sqrt{2} \approx 0.37$ as compared to the extrapolation of the high temperature result.

We arrive at the $H-T$ phase diagram shown in Fig. 1. The first order transition between the diamagnetic and the field penetration regime takes place between the spinodals $H_{s c} \sim \Phi_{o} / d^{2}<H_{b}(T)<H_{s h} \sim \Phi_{o} / \lambda_{N}(T)^{2}$ which delimit the (meta-)stable regime. Their intersection marks the critical temperature

$$
T_{\text {crit }} \approx T_{A} \log \left(d / \lambda_{N}\right)
$$

where $\lambda_{N}\left(T_{\text {crit }}\right) \approx d$. Below $T_{\text {crit }}$ the penetration depth is small, $\lambda_{N}\left(T_{\text {crit }}\right)<d$, and we observe a first order transition. Above the critical point $T_{\text {crit }}$, where $\lambda_{N}\left(T_{\text {crit }}\right)>d$, a continuous and reversible cross-over between the diamagnetic and field penetration regime is expected. We note that this distinction is similar to the one between Type I and Type II superconductors with respect to the penetration depth $\lambda$ and the superconducting coherence length $\xi$.

The latent heat (at $T \gg T_{A}$ ) of the transition follows from Eqs. (17), (18), and (20) using $S=-\partial \mathcal{G} / \partial T$,

$$
T \Delta S \approx \frac{3}{16 \pi} \frac{T}{T_{A}} H_{b}^{2}(T) d,
$$

and is related to the magnetization jump

$$
4 \pi \Delta \mathcal{M} \approx \frac{3}{4} H_{b}(T) d
$$

via the Clausius-Clapeyron equation.

In the derivation of the breakdown field we have used the asymptotic expansions of the free energies in $a / \Phi_{\circ}$ and $\Phi_{\circ} / a$, respectively. Their quality at the transition point is determined by the range of overlap between the diamagnetic and the field penetration regimes in Fig. 2, which is governed by the parameter $\lambda_{N}(T) / d$. In the diamagnetic phase, the corrections are of the order of $\left(a / \Phi_{\circ}\right)^{2} \sim\left(H_{b} \lambda_{N}^{2}(T) / \Phi_{\circ}\right)^{2} \sim\left(\lambda_{N}^{2}(T) / d\right)^{2}$, and similarly in the field penetration regime. The expansion thus breaks down at $\lambda_{N}(T) \approx d$, which is the critical point of the transition line. We note that the total magnetization changes from its diamagnetic value $\mathcal{M} \sim H_{b} d$ to the strongly suppressed value $\mathcal{M} \sim H_{b} d\left(\lambda_{N}(T) / d\right)^{2}$ at the transition, reflecting its strong first order character.

\section{EXPERIMENT}

Mota et al. 12, 13 have measured the breakdown field in $\mathrm{Ag}-\mathrm{Nb}$ cylinders. The clean limit theory valid for $T \gg v_{F} / l_{e l}$ may be used provided that $l_{e l} \gg d$, which is claimed to be fulfilled in the experiment. In our comparison we neglect the influence of diffusive boundary scattering or any potential barrier at the NS interface, and ignore the difference in geometry, cylindrical for the sample and planar in the theoretical model.

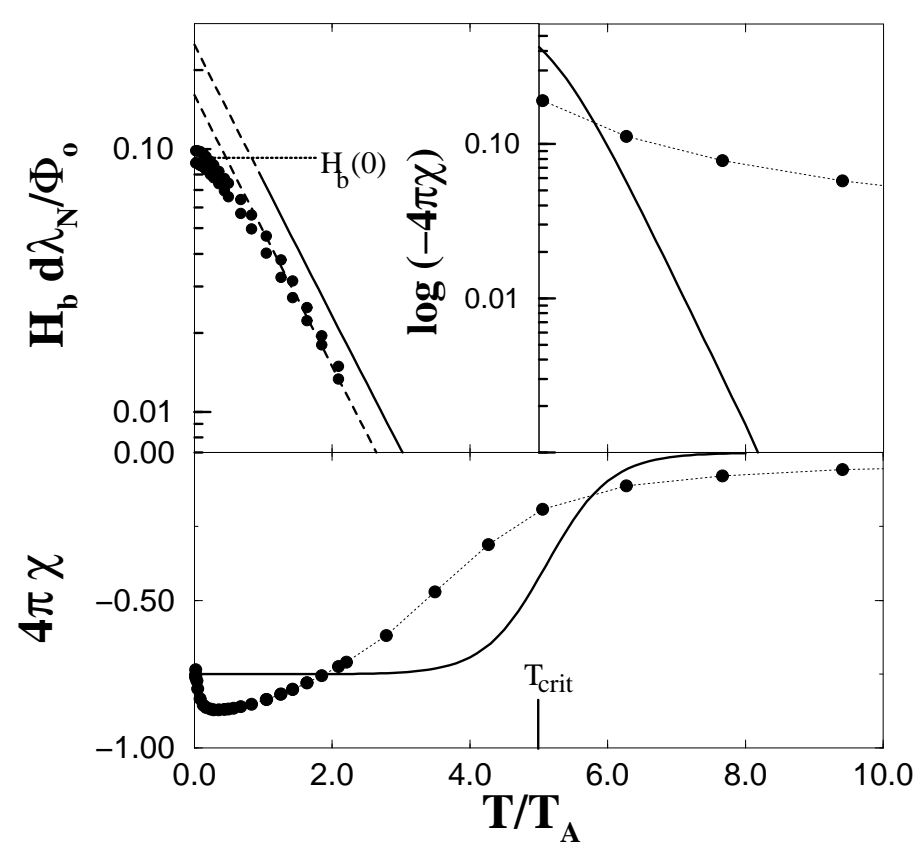

FIG. 3. Breakdown field $H_{b}(T)$ and linear susceptibility $4 \pi \chi$ from theory and experiment (the analysis applies to a Ag-Nb sample of thickness $d=5.5 \mu \mathrm{m})$. Theory: results of Eqs. (20) and (25) shown as solid lines; $H_{b}(T)$ is rescaled to fit the zero temperature value $H_{b}(0)$ (horizontal line) to the experiment. Experiment: data shown as solid dots, the dotted line is a guide to the eye [13]. Note that the logarithmic slope of the breakdown field is reproduced precisely (dashed line), while the one of the susceptibility is much smaller than expected.

In Fig. 3 we show the two data sets for the breakdown field data obtained on heating and cooling a sample of thickness $d=5.5 \mu \mathrm{m}$ exhibiting hysteresis (the theoretical values of the super-cooled and super-heated fields $H_{s c}$ and $H_{s h}$ are not reached in the experiments). The data saturates at low temperatures, in qualitative agreement with our theoretical analysis. Given the electron density in $\mathrm{Ag}, n=5.8 \cdot 10^{22}\left(\Rightarrow \lambda_{N}=2.2 \cdot 10^{-6}\right)$ and $d=5.5 \mu \mathrm{m}$, the breakdown field is determined by Eq. (19) and (20). Due to the idealization of our model, which assumes a step function for the order parameter, as well as the difference between the planar and cylindrical geometry, we expect a numerical factor correcting the amplitude of $H_{b}$. Making use of the scaling factor $\approx 0.56$ in Eqs. (19) and (20), we calibrate the theoretical result to fit the zero temperature value $H_{b}(0)$, as shown in Fig. 3. The theoretical prediction for the high temperature behavior then follows from Eq. (20) and is shown as a solid line in Fig. 3. Most importantly, Eq. (20) accurately reproduces the logarithmic slope $-1 / T_{A}$ of the experimental data, thus correctly tracing the signature of the Andreev 
levels. The amplitude of $H_{b}(T)$ deviates from the data by the constant ratio $\approx 0.64$, which can be attributed to the presence of a barrier at the NS interface, see below.

An important further agreement between theory and experiment is found in the scaling of the breakdown field with sample thickness $d$, which was reported to be $\propto 1 / d$, in accordance with Eq. (20) (the experimental study involved 10 samples 13, 16 with thicknesses ranging from $d=2.9 \mu \mathrm{m}$ to $d=28 \mu \mathrm{m})$. Similarly, the critical temperature determined in the experiment 12] exhibits the same scaling $\propto 1 / d$, in agreement with Eq. (21) $\left(T_{A} \propto 1 / d\right)$.

The only result in the literature on the breakdown field [6] was derived from the GL equations in the dirty limit $l_{e l} \ll \xi_{D}=\sqrt{v_{F} l_{e l} / 6 \pi T}<d$, with the coherence length $\xi_{D}$ limited by $\lambda_{D}(d) \ll \xi_{D}<d\left(\lambda_{D}(x)\right.$ is a space and temperature dependent penetration depth, see Ref. [6]). The breakdown field

$$
H_{D}(T) \approx 1.9 \frac{\Phi_{\circ}}{\lambda_{D}(0) \xi_{D}} \exp \left(-d / \xi_{D}\right)
$$

exhibits a simple exponential dependence on $d / \xi_{D} \propto \sqrt{T}$, the amplitude being temperature independent. Furthermore, no dependence of the amplitude on the thickness is present. Clearly, the experimental data deviates significantly from the predictions made by the GL theory.

The good agreement between the clean limit theory and experiment for the breakdown field does not trivially generalize to other physical quantities, however. In particular, the temperature dependence of the linear susceptibility $\chi=\mathcal{M} / H$ exhibits distinct features due to the non-locality of the constitutive relation $j(a)$ which are not observed in the experiment. From Eqs. (7) and (8) we obtain the susceptibility

$$
4 \pi \chi=\frac{4 \pi \mathcal{M}}{H}=-\frac{3}{4} \frac{1}{1+3 \lambda_{N}^{2}(T) / d^{2}},
$$

which exhibits a temperature dependence much like a Fermi-Dirac distribution: $4 \pi \chi$ decays exponentially $\propto$ $1 / \lambda_{N}^{2}(T)$ at large temperatures, twice as fast as the breakdown field. The susceptibility takes half its maximal value at $3 \lambda_{N}^{2}\left(T_{1 / 2}\right) / d^{2} \sim 1$, which roughly coincides with the critical temperature $T_{\text {crit }}$ (see Fig. 3). Below the critical point, the susceptibility saturates as the penetration depth decreases below the sample thickness $\left(\lambda_{N}(T)<d\right)$. Due to the non-locality, the penetration depth drops out of the expression for $4 \pi \chi \approx-3 / 4$ and we are in the regime of over-screening. The logarithmic derivative at $T=T_{1 / 2}$ is predicted to be $\chi^{\prime}\left(T_{1 / 2}\right) / \chi\left(T_{1 / 2}\right)=1 / T_{A}$. In Fig. 3 we show the linear susceptibility according to the clean limit predictions (25) (there is no fitting parameter). The experimental data fails to show the typical saturation of the susceptibility expected below the critical temperature. At low temperature the experimental value clearly exceeds the maximal diamagnetic value $-3 / 4$ found in the clean limit (note that we do not consider the anomalous re-entrance effect of these samples at very low temperatures here). Most strikingly, the decay at large temperature is slower than the decay of the breakdown field, while Eq. (25) predicts a decay with twice the logarithmic slope, see Fig. 3 . Thus the magnetic behavior of the quasi-ballistic samples deviates from the clean limit theory, indicating that the elastic mean free path is of order of the thickness of the sample. We find that the susceptibility emerges as a very sensitive indicator of the non-locality of the constitutive relations.

Let us address the question whether the consideration of a insulating barrier at the NS interface may lift the discrepancy. The consequences of a finite reflectivity at the NS interface on the linear current response has been analyzed by Higashitani and Nagai [17. Their results allow for the reflection coefficient $R$ to be included in the penetration depth $\lambda_{N}(T)$ by redefining the factor $\gamma_{R}\left(T_{A} \ll T \ll \Delta\right)=(1-R) /(1+R)$, in Eq. (16); $\lambda_{N}(0) \equiv \lambda_{N}$ remains unchanged [17. Inserting the modified penetration depth into Eq. (25) we obtain the linear susceptibility. The additional factor $\gamma$ does not change the characteristic shape of the susceptibility (saturation, logarithmic slope at $T_{1 / 2}$, exponential decay), but only lowers the position of the half-value of $\chi$ to $T_{1 / 2} \approx \log \left[d(1-R) / \lambda_{N}(1+R)\right]$. Thus the finite reflection does not remedy the qualitative discrepancy between theory and experiment, which may have to be attributed to disorder or diffusive boundary scattering.

Considering the structure of the equations we may expect the dependence on the reflection $R$ to enter in a similar fashion into the breakdown field $H_{b}(T)$, although we note that this has not been shown rigorously. Eq. (25) inserted in Eq. (20) gives the high temperature behavior, while the zero temperature result of Eq. (19) remains unchanged. We fit the breakdown field data by using first an overall scaling factor needed to adjust $H_{b}(0)$ and secondly, a finite reflectivity, which only enters at high temperatures. The fit of the high temperature behavior provides us with an estimate of the reflectivity $R \approx 0.21$, and is represented by the dashed line in Fig. 3 .

In conclusion, we have calculated the clean limit expression for the breakdown field separating the diamagnetic phase and the field penetration phase by a first order transition. We have determined the spinodals, the critical temperature as well as the latent heat of the transition. In comparison with the experimental data on quasi-ballistic samples, we have found good agreement with respect to the dependences on temperature and thickness of $H_{b}(T, d)$ and $T_{\text {crit }}(d)$. The inclusion of a finite reflection at the NS interface permits an accurate fit of the breakdown field and gives an estimate for the quality of the NS interface. However, with regard to the linear susceptibility, the experiments disagree with the clean limit theory, showing the need to include additional scattering processes. The susceptibility thus emerges as 
a quantity which is very sensitive to the non-locality of the constitutive relations.

We are indebted to V. Geshkenbein for the frequent, elucidating discussions throughout this work. We gratefully acknowledge A.C. Mota and B. Müller for the discussion of their experimental data. We have also benefited from a stimulating exchange with W. Belzig, C. Bruder, G. Lesovik, M. Sigrist, and A. Zaikin.

[1] A recent review is found in Mesoscopic Electron Transport, eds. L.P. Kouwenhoven, G. Schön, and L.L. Sohn, NATO ASI Series E (Kuwer Academic Publ. Dortrecht) to be published.

[2] Y. Oda and H. Nagano, Solid State Comm. 35, 631 (1980).

[3] A.C. Mota, D. Marek, and J.C. Weber, Helv. Phys. Acta 55, 647 (1982).

[4] Th. Bergmann, K.H. Kuhl, B. Schroeder, M. Jutzler, and
F. Pobell, J. Low Temp. Phys. 66, 209 (1987).

[5] P. Visani, A.C. Mota, and A. Pollini, Phys. Rev. Lett. 65, 1514 (1990).

[6] Orsay Group on Superconductivity, in Quantum Fluids, p. 26, North-Holland (1966).

[7] For a derivation see A.L. Shelankov, J. Low. Temp. Phys. 60, 29 (1985).

[8] G. Eilenberger, Z. Phys. 214, 195 (1968).

[9] K.D. Usadel, Phys. Rev. Lett. 25, 507 (1970).

[10] A.D. Zaikin, Solid State Comm. 41, 533 (1982).

[11] W. Belzig, C. Bruder, G. Schön, Phys. Rev. B 53, 5727 (1996).

[12] A.C. Mota, P. Visani, and A. Pollini, J. Low Temp. Phys. 76, 465 (1989).

[13] A.C. Mota, P. Visani, A. Pollini, K. Aupke, Physica B 197, (1994).

[14] Neglecting the fields in the superconductor permits the choice of gauge $A(0)=0$.

[15] The numerical data has kindly been provided by W. Belzig, Universität Karlsruhe.

[16] We acknowledge unpublished data provided by B. Müller and A. Mota, ETH Zürich.

[17] S. Higashitani, K. Nagai, J. Phys. Soc. J. 64, 549 (1995). 